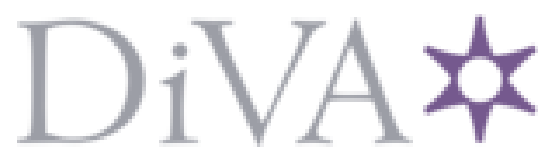

http://www.diva-portal.org

This is the published version of a paper presented at 5th IFAC Symposium on Nonlinear Control Systems, JUL 04-06, 2001, ST PETERSBURG, RUSSIA.

Citation for the original published paper:

Freidovich, L., Khalil, H. (2001)

Universal integral controllers for robotic manipulators.

In: NONLINEAR CONTROL SYSTEMS 2001, VOLS 1-3 (pp. 351-356).

IFAC SYMPOSIA SERIES

N.B. When citing this work, cite the original published paper.

Permanent link to this version:

http://urn.kb.se/resolve?urn=urn:nbn:se:umu:diva-88145 


\title{
UNIVERSAL INTEGRAL CONTROLLERS FOR ROBOTIC MANIPULATORS ${ }^{1}$
}

\author{
Leonid B. Freidovich* , Hassan K. Khalil** \\ * Department of Mathematics, \\ Michigan State University, \\ East Lansing, MI 48824, USA \\ e-mail: leonid@math.msu.edu \\ ** Department of Electrical and Computer Engineering, \\ Michigan State University, \\ East Lansing, MI 48824, USA \\ e-mail:khalil@msu.edu
}

\begin{abstract}
As a standard model for a rigid multilink robotic manipulator, we consider a MIMO nonlinear system of uniform vector relative degree $\{2, \ldots, 2\}$, which has a globally defined normal form with no zero dynamics. We consider a trajectoryfollowing problem for a class of smooth bounded time-varying vector reference signals. We design an output feedback integral controller that ensures ultimately bounded tracking error which could be made as small as required. In addition, if the reference signal has a constant limit, the output approaches this limit. The controller is robust with respect to parameter uncertainties, decentralized, saturated, and simple to implement. Locally, it is a PID regulator with derivatives estimated via a linear highgain observer. Regional (and semiglobal) practical (and asymptotic) stability is shown with the help of a vector Lyapunov function and a technique, typical for "continuous" sliding mode control.
\end{abstract}

Keywords: Robot Control, Saturated Control, PID Control, Ultimate Boundedness, Semiglobal Asymptotic Stability, High-gain Observers

\section{INTRODUCTION}

The regulation problem for directly controlled mechanical systems and, in particular, rigid robotic manipulators has attracted attention of many specialists in modern control theory. A lot of powerful results using different stabilization techniques are available today. Nevertheless, most industrial robots are controlled through simple saturated proportional-integral-derivative (PID) like regulators, typically with on-line derivative

1 This work was supported in part by the USA National Science Foundation under Grant no. ECS-9703742 estimators. Thus, there is a gap between the theoretical results available and the common industrial practice. Saturated regulators studied in (Burkov, 1995; Dunskaya and Pyatnitskii, 1988; Freidovich, 1999; Loria et al., 1996) and output regulators proposed in (Berghuis and $\mathrm{Ni}$ jmeijer, 1993; Burkov, 1995; Kelly, 1998; Loria et al., 1996) require some precise information on model parameters and structure. Local, regional, and semiglobal stabilization results for state PID and PID-like regulators are shown in (Alvarez-Ramirez et al., 2000; Arimoto and Miyazaki, 1984; Freidovich, 1999; Kelly, 1998; 
Meressi et al., 1993; Ortega et al., 1998; Pervozvanski and Freidovich, 1999; $\mathrm{Qu}$ and Dorsey, 1991; Rocco, 1996; Takegaki and Arimoto, 1981). Partially saturated state PID-like regulators of (Arimoto, 1996; Gorez, 1999; Kelly, 1998; Santibañez and Kelly, 1998; Tomei, 1991) guarantee global asymptotic stability. Semiglobally stabilizing robust output PID-like regulators were addressed in (Ortega et al., 1998; Pervozvanski and Freidovich, 1999). We discuss these results in the last section of this paper. So far, to the best of our knowledge, the only output saturated globally stable PID-like regulation result available is the one of (Gorez, 1999), but the regulator is not simple at all and requires some hard to obtain friction parameter estimates for tuning.

The main contribution of this paper is a semiglobally stabilizing saturated output decentralized PID controller, with derivatives estimated through a linear high-gain observer, to track arbitrary asymptotically constant smooth reference signals. If there is no constant limit but the reference signal is bounded with two bounded derivatives, ultimate boundedness of the tracking error is shown. The controller is a generalization of the universal integral regulator for a minimum phase SISO nonlinear systems (Khalil, 2000), to the case of a uniform vector relative degree $\{2, \ldots, 2\}$ multiinput multi-output (MIMO) system in normal form with no zero dynamics and positive definite decoupling matrix uniformly bounded from above and below. Structurally, our regulator is a combination of a continuous approximation of a discontinuous sliding mode controller and a highgain linear observer. Inside the domain where the controller is not saturated and the observation error is negligible, it is equivalent to a high-gain linear decentralized PID regulator.

\section{DYNAMICAL MODEL AND CONTROL DESIGN}

The dynamics of rigid robotic manipulators can be described by the following system of first order differential equations (Spong and Vidyasagar, 1989)

$$
A(q, \theta) \dot{v}+C(q, v, \theta) v+g(q, \theta)=u, \quad \dot{q}=v,
$$

where $q \in \mathbf{R}^{n}$ is the vector of generalized coordinates (link positions); $v \in \mathbf{R}^{n}$ is the vector of generalized velocities; $\theta$ is the vector of unknown parameters that belong to a given compact set $\Theta \in \mathbf{R}^{l} ; A(q, \theta)$ is the positive definite robot inertia matrix, and we assume (Spong and Vidyasagar, 1989; Ortega et al., 1998) that there are $m>0$ and $M>0$ such that $\forall q \in \mathbf{R}^{n}, \theta \in \Theta$

$$
m I \leq A(q, \theta) \leq M I
$$

( $I$ is the identity matrix); $C(q, v, \theta) v$ is the vector of Coriolis and centrifugal forces; $g(q, \theta)$ is the vector of gravitational forces; and $u$ is the vector of control torques. We assume that the only information available on-line is the output vector $y=q(t)$, that is the vector of the manipulator link positions.

The goal is to design a robust saturated output dynamic controller to ensure that the output $y(t)$ asymptotically tracks a reference signal $r(t)$ with an ultimately bounded error that can be made as small as required, provided the initial conditions belong to a given compact set.

We assume that $r(t)$ is a twice continuously differentiable vector-function, such that $\|r(t)\|$, $\|\dot{r}(t)\|$, and $\|\ddot{r}(t)\|$ are bounded by à priori known constants.

Also, we show that if there exists a constant vector $q_{d}$, such that $\lim _{t \rightarrow \infty}\left\|r(t)-q_{d}\right\|=0$ and $q_{d}$ belongs to a given compact set $W_{d} \subset \mathbf{R}^{n}$ and, moreover, $\lim _{t \rightarrow \infty}\|\dot{r}(t)\|=0, \lim _{t \rightarrow \infty}\|\ddot{r}(t)\|=0$, then $\lim _{t \rightarrow \infty}\left\|y(t)-q_{d}\right\|=0$.

Let $\nu^{T}(t)=\left[\left(r(t)-q_{d}\right)^{T}, \dot{r}^{T}(t), \ddot{r}^{T}(t)\right]$ (if there is no constant limit, take $q_{d}=0$ and $W_{d}=\{0\}$ ), then there exists an à priori known compact set $\Lambda \subset \mathbf{R}^{3 n}$ such that $\nu(t) \in \Lambda, \forall t$. Also, let $d^{T}=\left[q_{d}^{T}, \theta^{T}\right]$, then $d \in D=W_{d} \times \Theta \subset \mathbf{R}^{n+l}$.

To write the error equation, let us introduce the following notation

$$
\begin{gathered}
e^{T}=\left[e_{1}^{T}, e_{2}^{T}\right]=\left[(q-r)^{T},(v-\dot{r})^{T}\right], \\
a_{0}(e, \nu, d)=A^{-1}\left(e_{1}+r, \theta\right), \\
b_{0}(e, \nu, d)=-a_{0}(e, \nu, d)\left[C ( e _ { 1 } + r , e _ { 2 } + \dot { r } , \theta ) \left(e_{2}+\right.\right. \\
\left.\dot{r})+g\left(e_{1}+r, \theta\right)\right]-\ddot{r} .
\end{gathered}
$$

Then (1) can be written as

$$
\dot{e}_{1}=e_{2}, \quad \dot{e}_{2}=b_{0}(e, \nu, d)+a_{0}(e, \nu, d) u,
$$

which is a (global) normal form with no zero dynamics for a nonlinear MIMO system of uniform vector relative degree $\{2, \ldots, 2\}$ (Isidori, 1995).

We augment this system with an integrator driven by the tracking error

$$
\dot{\sigma}=e_{1}
$$

and, by analogy with (Khalil, 2000), take the controller as

$$
u=-K \operatorname{sat}\left(\mu^{-1} \hat{s}\right),
$$

i.e. $\quad u_{i}=-k_{i} \operatorname{sat}\left(\hat{s}_{i} / \mu_{i}\right) \quad$ for $\quad i=1 \ldots n$. Here "sat" is the standard saturation function (Khalil, 1996, p.73); $K=\operatorname{diag}\left\{k_{i}\right\}_{i=1}^{n} ; \quad k_{i}>0$ 
are saturation levels; $\mu=\operatorname{diag}\left\{\mu_{i}\right\}_{i=1}^{n} ; \mu_{i}>0$ are small parameters ( $k_{i} / \mu_{i}$ are local high gains);

$$
\hat{s}=K_{1} \sigma+K_{2} e_{1}+\hat{e}_{2},
$$

where $K_{1}=\operatorname{diag}\left\{k_{1 i}\right\}_{i=1}^{n}$ and $K_{2}=\operatorname{diag}\left\{k_{2 i}\right\}_{i=1}^{n}$ with $k_{1 i}>0$ and $k_{2 i}>0$, so that $p^{2}+k_{2 i} p+k_{1 i}$ are Hurwitz; $\hat{e}_{2}$ is the output of the full-order high-gain observer

$$
\dot{\hat{e}}_{1}=\hat{e}_{2}+\frac{\alpha_{1}}{\varepsilon}\left(e_{1}-\hat{e}_{1}\right), \quad \dot{\hat{e}}_{2}=\frac{\alpha_{2}}{\varepsilon^{2}}\left(e_{1}-\hat{e}_{1}\right),
$$

where $\alpha_{1}>0$ and $\alpha_{2}>0$ (so that $p^{2}+\alpha_{1} p+\alpha_{2}$ is Hurwitz) and $\varepsilon>0$ is a small parameter (the bandwidth of the high-gain observer is proportional to $1 / \varepsilon$ ).

\section{STABILITY ANALYSIS}

Let us rewrite the closed-loop system (2),(3),(4), (5) in a form more convenient for analysis:

$$
\begin{aligned}
& \dot{\zeta}=A \zeta+B s \\
& \left.\dot{s}=\Delta(\cdot)-a_{0}(\cdot) K \operatorname{sat}\left(\mu^{-1} \hat{s}\right)\right) \\
& \varepsilon \dot{\varphi}=A_{f} \varphi+\varepsilon B\left(b_{0}(\cdot)-a_{0}(\cdot) K \operatorname{sat}\left(\mu^{-1} \hat{s}\right)\right)
\end{aligned}
$$

where

$$
\begin{gathered}
s=K_{1} \sigma+K_{2} e_{1}+e_{2}=\hat{s}+\left(e_{2}-\hat{e}_{2}\right), \quad B=\left[\begin{array}{l}
0 \\
I
\end{array}\right], \\
\varphi=\left[\begin{array}{l}
\varphi_{1} \\
\varphi_{2}
\end{array}\right]=\left[\begin{array}{c}
\varepsilon^{-1}\left(e_{1}-\hat{e}_{1}\right) \\
e_{2}-\hat{e}_{2}
\end{array}\right], \quad \zeta=\left[\begin{array}{c}
\sigma \\
e_{1}
\end{array}\right], \\
A=\left[\begin{array}{cc}
0 & I \\
-K_{1} & -K_{2}
\end{array}\right], \quad A_{f}=\left[\begin{array}{cc}
-\alpha_{1} I & I \\
-\alpha_{2} I & 0
\end{array}\right], \\
\Delta(e, \nu, d)=b_{0}(e, \nu, d)+K_{1} e_{1}+K_{2} e_{2} .
\end{gathered}
$$

Let us show that trajectories of the closed-loop system with initial conditions in a given compact set enter a small positively invariant set in finite time and that this set could be made arbitrarily small, provided $\mu_{i}$ 's can be chosen small enough.

After this, for the case of asymptotically constant reference signals, we show that the trajectories, trapped inside the invariant set, must approach a unique equilibrium point. Hence, asymptotic tracking is guaranteed for finite gains.

Obviously, $A$ and $A_{f}$ are Hurwitz matrices, hence there exist positive definite matrices $P$ and $P_{f}$, which satisfy the Lyapunov equations

$$
P A+A^{T} P=-I \text { and } P_{f} A_{f}+A_{f}^{T} P_{f}=-I .
$$

In the proofs below we use the three Lyapunov functions

$V=\zeta^{T} P \zeta, \quad V_{f}=\varphi^{T} P_{f} \varphi, \quad W(s, \zeta)=s^{T} A(\cdot) s / 2$ (note: $\forall \zeta \in \mathbf{R}^{2 n}: m\|s\|^{2} / 2 \leq W \leq M|| s \|^{2} / 2$ ) and the sets: $\Omega_{\zeta}(R)=\left\{\zeta \in \mathbf{R}^{2 n}: V(\zeta) \leq \rho_{1} R^{2}\right\}$,

$$
\begin{aligned}
& \Omega_{s}(R)=\left\{s \in \mathbf{R}^{n}: \exists \zeta \quad \text { s.t. } W(\zeta, s) \leq R^{2} / 2\right\}, \\
& \Omega(R)=\left\{(\zeta, s) \in \mathbf{R}^{3 n}: V \leq \rho_{1} R^{2}, W \leq R^{2} / 2\right\}, \\
& \text { and } \quad \Sigma_{\varepsilon}(R)=\left\{\varphi \in \mathbf{R}^{2 n}: V_{f}(\varphi) \leq \varepsilon^{2} \rho_{2}(R)\right\},
\end{aligned}
$$

where $\rho_{1}=\|P\| \gamma_{0}^{2} / m, \quad \rho_{2}(R)=\left\|P_{f}\right\| \gamma_{2}^{2}(R)$, $\gamma_{0}=4|| P B \|$, and $\gamma_{2}(R)=4|| P_{f} B \| \sup \left\{\| b_{0}(\cdot)-\right.$ $a_{0}(\cdot) K \operatorname{sat}\left(\mu^{-1} \hat{s}\right) \|: d \in D, \nu \in \Lambda, \varphi \in \mathbf{R}^{2 n},(\zeta, s)$ $\in \Omega(R)\}$.

Let also $\gamma_{1}(R)=\operatorname{diag}\left\{\gamma_{1 i}(R)\right\}_{i=1}^{n}$ be such that $\forall d \in D, \nu \in \Lambda, \varphi \in \mathbf{R}^{2 n},(\zeta, s) \in \Omega(R)$ :

$$
s^{T} A(\cdot) \Delta(\cdot)+\frac{1}{2} s^{T} \dot{A}(\cdot) s \leq \sum_{i=1}^{n} \gamma_{1 i}(R)\left|s_{i}\right|
$$

Theorem 1. Suppose that the initial conditions for the closed-loop system (2),(3),(4), (5) belong to a given compact set. There exist $c>0, \bar{\mu}>0$ and $\bar{\varepsilon}>0$ such that for all $\varepsilon \in(0, \bar{\varepsilon}], \mu:\|\mu\| \in$ $(0, \bar{\mu}]$, and $K>\gamma_{1}(c)$ the trajectories enter some small (shrinking as $\|\mu\| \rightarrow 0$ ) compact set, in finite time, and stay there for all future times.

Proof. To show Lagrange stability and then practical stability we use sliding mode control ideas for the $s$-subsystem, exponential input-tostate stability of the $\zeta$-subsystem driven by $s$, and exponential input-to-state stability of the $\varphi$ subsystem driven by $s$ and $\zeta$.

It is not hard to check that $\forall(\zeta, s, \varphi) \in \Omega(R) \times$ $\Sigma_{\varepsilon}(R)$ the following inequalities hold

$$
\begin{gathered}
\dot{V} \leq-\frac{1}{2}\|\zeta\|^{2}-\frac{\|\zeta\|}{2}\left(\|\zeta\|-\gamma_{0}\|s\|\right), \\
\dot{W} \leq-s^{T} K \operatorname{sat}\left(\mu^{-1} \hat{s}\right)+\sum_{i=1}^{n} \gamma_{1 i}(R)\left|s_{i}\right|= \\
-\sum_{i=1}^{n}\left|s_{i}\right|\left(k_{i}\left|\operatorname{sat}\left(\mu_{i}^{-1} \hat{s}_{i}\right)\right|-\gamma_{1 i}(R)\right), \\
\dot{V}_{f} \leq-\frac{1}{2 \varepsilon}\|\varphi\|^{2}-\frac{\|\varphi\|}{2 \varepsilon}\left(\|\varphi\|-\varepsilon \gamma_{2}(R)\right) .
\end{gathered}
$$

The rest of the analysis proceeds in three steps.

(1) Obviously, for a given compact set of initial conditions, there exists $c>0$ such that initially $(\zeta, s)$ belong to some compact set strictly inside $\Omega(c)$. Assume that initially $\varphi \in \Sigma_{\varepsilon}(c)$ for some small $\varepsilon>0$. A trajectory may leave the set $\Omega(c) \times \Sigma_{\varepsilon}(c)$ only through one of the boundaries: $V(\cdot)=\rho_{1} c^{2}$, $W(\cdot)=c^{2} / 2$, or $V_{f}(\cdot)=\varepsilon^{2} \rho_{2}(c)$. Let us show that it is impossible.

(a) $V_{f}(\varphi)=\varphi^{T} P_{f} \varphi=\varepsilon^{2} \rho_{2}(c)$ implies

$$
\|\varphi\| \geq \sqrt{\varepsilon^{2} \rho_{2}(c)\left\|P_{f}\right\|^{-1}}=\gamma_{2}(c) \varepsilon .
$$


Hence $\quad \dot{V}_{f} \leq-\frac{1}{2 \varepsilon}\|\varphi\|^{2} \leq-\frac{c_{1}}{\varepsilon} V_{f}<0$. So, $V_{f}(\varphi(t))$ is a decreasing function of $t$ and $\varphi(t)$ stays in $\Sigma_{\varepsilon}(c)$, where

$$
\begin{gathered}
\|\varphi(t)\| \leq \varepsilon \rho_{3}(c) \\
\text { and } \rho_{3}(c)=\gamma_{2}(c) \sqrt{\left\|P_{f}\right\|\left\|P_{f}^{-1}\right\|} \\
\text { (b) } W(s, \zeta)=s^{T} A(\cdot) s / 2=c^{2} / 2 \text { implies }
\end{gathered}
$$

$$
\|s\| \geq c / \sqrt{M} .
$$

Hence, assuming that $c / \sqrt{M}>(n-$ 1) $\left(\|\mu\|+\varepsilon \rho_{3}(c)\right)$ one can show that $\left|s_{i}\right|>$ $\mu_{i}+\varepsilon \rho_{3}(c)$ for at least one index $i \in$ $\{1, \ldots, n\}$ for each $s$. Using the following notation

$N^{+}(s)=\left\{i \in\{1, \ldots, n\}:\left|s_{i}\right| \geq \mu_{i}+\varepsilon \rho_{3}(c)\right\}$, $N^{-}(s)=\left\{i \in\{1, \ldots, n\}:\left|s_{i}\right|<\mu_{i}+\varepsilon \rho_{3}(c)\right\}$,

and the definition of the saturation function, one may easily derive that

$$
\begin{aligned}
& \dot{W} \leq-\sum_{i \in N^{+}(s)}\left(k_{i}-\gamma_{1 i}(c)\right)\left|s_{i}\right|+\sum_{i \in N^{-}(s)}\left(\gamma_{1 i}(c)\left|s_{i}\right|\right. \\
& \left.-k_{i}\left|s_{i}\right|\left(\frac{\left|s_{i}\right|}{\mu_{i}+2 \varepsilon \rho_{3}(c)}-\frac{\left|\varphi_{2 i}\right|}{\mu_{i}}\right)\right) .
\end{aligned}
$$

Using the estimate for the maximum of a concave quadratic function

$$
\left|s_{i}\right|\left(\tilde{\gamma}_{1 i}(c)-\frac{k_{i}}{\tilde{\mu}_{i}(c)}\left|s_{i}\right|\right) \leq \frac{\tilde{\mu}_{i}(c) \tilde{\gamma}_{1 i}^{2}(c)}{4 k_{i}}
$$

where $\tilde{\gamma}_{1 i}(c)=\gamma_{1 i}(c)+\varepsilon \rho_{3}(c) k_{i} / \mu_{i}$ and $\tilde{\mu_{i}}(c)=\mu_{i}+2 \varepsilon \rho_{3}(c)$, it is not hard to show that $\dot{W} \leq-c_{2}$, where

$c_{2}=\min _{i}\left\{k_{i}-\gamma_{1 i}(c)\right\}(c / \sqrt{M}-(n-1)(\|\mu\|$ $\left.\left.+\varepsilon \rho_{3}(c)\right)\right)-(n-1) \max _{i}\left\{\tilde{\mu}_{i}(c) \tilde{\gamma}_{1 i}^{2}(c) / k_{i}\right\} / 4$.

Hence, for $\|\mu\|$ and $\varepsilon$ small enough, $c_{2}$ is positive and, correspondingly, $\dot{W}<$ 0 . Then, $W(s(t), \zeta(t))$ is a decreasing function of $t$, so $s(t)$ stays in $\Omega_{s}(c)$ and

$$
\|s(t)\| \leq c / \sqrt{m}
$$

(c) $V(\zeta)=\zeta^{T} P \zeta=c^{2} \rho_{1}$ implies

$$
\|\zeta\| \geq \sqrt{\rho_{1} c^{2} /\|P\|}=\gamma_{0} c / \sqrt{m} \geq \gamma_{0}\|s\|
$$

and, hence, $\dot{V} \leq-\frac{1}{2}\|\zeta\|^{2} \leq-c_{3} V<0$. So, $V(\zeta(t))$ is a decreasing function of $t$ and $\zeta(t)$ stays in $\Omega_{\zeta}(c)$, where $\|\zeta(t)\| \leq$ $c \gamma_{0} \sqrt{\|P\|\left\|P^{-1}\right\| / m}$.

Thus, the set $\Omega(c) \times \Sigma_{\varepsilon}(c)$ is positively invariant, i.e. the trajectories of (6) stay in it once they have entered it.

(2) In the beginning of the previous step we have assumed that $\varphi(0) \in \Sigma_{\varepsilon}(c)$, which is too restrictive. If the initial conditions for (5), $\hat{e}(0)$, are not close enough to the initial conditions for $(2), e(0)$, then ||$\varphi(0) \mid$ is pretty large, in fact, of the order of $1 / \varepsilon$.

In this case, the system (6) is not in standard singular perturbed form, hence Tikhonov's theorem (Khalil, 1996, p.361) and the result of (Hoppensteadt, 1974) are not applicable.

Take $\hat{e}(0)$ in a compact set $\Phi$. Let

$$
\Phi_{\varepsilon}(c)=\left\{\varphi \in \mathbf{R}^{2 n}:(\zeta, s) \in \Omega(c), \hat{e} \in \Phi\right\},
$$

then $\varphi(0) \in \Phi_{\varepsilon}(c)$. Obviously, there exists $b$ such that $0<b<c$ and that initially $(\zeta, s, \varphi) \in \Omega(b) \times \Phi_{\varepsilon}(c)$.

Using the estimates of (1a), it can be shown that for $\varepsilon$ small enough, $\varphi(t)$ enters the set $\Sigma_{\varepsilon}(c)$ in time smaller then $T(\varepsilon)=$ $\varepsilon\left(c_{4}-c_{5} \ln \varepsilon\right.$ ) (Atassi and Khalil, 1999). During this short period of time the peaking phenomenon occurs. Boundedness, uniformly in $\varepsilon$, of the right-hand side of the $s$-subsystem and continuous dependence of the solutions of (2) with respect to changes in $u$ allow us to claim that $(\zeta(t), s(t))$ will not change much and, therefore, will stay inside $\Omega(c)$.

Hence, for any given set of initial conditions for the closed-loop system (2), (3),(4), (5), the corresponding trajectories of (6) enter the set $\Omega(c) \times \Sigma_{\varepsilon}(c)$ in finite time.

(3) Let $\delta$ be a positive number. Our last task is to show that the trajectories enter the set $\Omega(\delta\|\mu\|) \times \Sigma_{\varepsilon}(c)$ in finite time and stay there for all future times.

(a) According to the previous step, $\varphi(t)$ enters $\Sigma_{\varepsilon}(c)$ and, according to (1a), cannot leave it.

(b) $\zeta \in \Omega_{\zeta}(c) \backslash \Omega_{\zeta}(\delta\|\mu\|)$ implies $W(s, \zeta) \geq$ $(\delta|| \mu||)^{2} / 2$ and

$$
\|s\| \geq \delta|| \mu \| / \sqrt{M}
$$

If $N^{+}(s) \neq \emptyset$ then, it could be shown similar to (1b), that if $\delta$ is large enough so that

$$
\delta|| \mu \| / \sqrt{M}>(n-1)\left(\|\mu\|+\varepsilon \rho_{3}(c)\right) \quad \text { and }
$$$$
\min _{i}\left\{k_{i}-\gamma_{1 i}(c)\right\}(\delta\|\mu\| / \sqrt{M}-(n-1)(\|\mu\|
$$$$
\left.\left.+\varepsilon \rho_{3}(c)\right)\right)>(n-1) \max _{i}\left\{\tilde{\mu}_{i}(c) \tilde{\gamma}_{1 i}^{2}(c) / k_{i}\right\} / 4 \text {, }
$$

then $\dot{W} \leq-c_{6}<0$. If $N^{+}(s)=\emptyset$ then none of the components of $u$ are saturated and one obtains

$$
\begin{gathered}
\dot{W} \leq-\sum_{i=1}^{n}\left(\frac{k_{i}}{\mu_{i}} s_{i}^{2}-\tilde{\gamma}_{1 i}(c)\left|s_{i}\right|\right) \leq-\frac{\|s\|^{2}}{2\left\|\mu K^{-1}\right\|} \\
-\frac{\|s\|^{2}}{2\left\|\mu K^{-1}\right\|}\left(|| s\left\|-\bar{\gamma}_{1}(c)\right\| \mu\|\| K^{-1} \|\right), \\
\text { where } \bar{\gamma}_{1}(c)=2 \sqrt{n}\left\|\tilde{\gamma}_{1}(c)\right\| .
\end{gathered}
$$

Assume that $\delta$ is large enough, so that

$$
\delta / \sqrt{M} \geq \bar{\gamma}_{1}(c)|| K^{-1} \| .
$$


Then either $\dot{W} \leq-c_{6}<0$ or $\dot{W} \leq$ $-c_{7} W<0$, and, hence, $W(\zeta(t), s(t))$ is a decreasing function of $t$. So, $s(t)$ enters the set $\Omega_{s}(\delta\|\mu\|)$ in finite time and stays there for all future times.

(c) Following the arguments presented in (1c), it is not hard to see that, once $s(t)$ is in the set $\Omega_{s}(\delta\|\mu\|), \quad \zeta(t)$ enters the set $\Omega_{\zeta}(\delta\|\mu\|)$ in finite time and stays in it for all future times.

Hence, the set $\Omega(\delta\|\mu\|) \times \Sigma_{\varepsilon}(c)$ is positively invariant and attracting for all trajectories of the system (6) originating inside the set $\Omega(b) \times \Phi_{\varepsilon}(c)$.

Consider now the case when the reference signal approaches a given constant $q_{d}$. To prove asymptotic stability of the closed-loop system, we show: that the trajectories enter an invariant set $\Omega(\delta|| \mu||) \times \Sigma_{\varepsilon}(c)$ for sufficiently small $\delta$, that the system is locally asymptotically stable, and that the region of attraction for (6), with "sat" substituted with the corresponding linear function, does not shrink as $\varepsilon \rightarrow 0$ and $\|\mu\| \rightarrow 0$.

We need $\delta>0$ to be so small, that inside the attracting set the control law (4), (5) is a linear dynamic decentralized one. Then it can be written using transfer function notation in the following form:

$u_{i}=-\left[\left(\frac{k_{i} k_{1 i}}{\mu_{i}}\right) \frac{1}{p}+\left(\frac{k_{i} k_{2 i}}{\mu_{i}}\right)+\left(\frac{k_{i}}{\mu_{i}} h(p)\right) p\right] e_{1 i}$

where $h(p) p=\left(\alpha_{2} p\right) /\left(\varepsilon^{2} p^{2}+\alpha_{1} \varepsilon p+\alpha_{2}\right)$ is the transfer function of the observer, which is an approximate differentiator.

Theorem 2. Suppose $\nu(t) \rightarrow 0$ as $t \rightarrow \infty$,

$$
\begin{aligned}
& \min _{i}\left\{\mu_{i}\left(k_{i}-\gamma_{1 i}(c)\right)\right\} \geq(n-1) \max _{i}\left\{\frac{\tilde{\mu}_{i}(c) \tilde{\gamma}_{1 i}^{2}(c)}{4 k_{i}}\right\}, \\
& \min _{i} k_{i} \geq 2 \sqrt{M n}\left\|\tilde{\gamma}_{1}(c)\right\| / \delta
\end{aligned}
$$

where $\delta>0$ is such that

$$
\varepsilon \rho_{3}(c)+\delta\left(\max _{i} \mu_{i}\right) / \sqrt{m} \leq \min _{i} \mu_{i}
$$

and, additionally, $\varepsilon$ and $\mu_{i}$ 's are sufficiently small. Then the trajectories approach the equilibrium point

$$
(\zeta, s, \varphi)=(\bar{\zeta}, \bar{s}, 0)=\left(\left[\bar{\sigma}^{T}, 0^{T}\right]^{T}, K_{1} \bar{\sigma}, 0\right),
$$

where $\bar{\sigma}=K_{1}^{-1} \mu K^{-1} g\left(q_{d}, \theta\right)$. Moreover, if $\nu(t) \equiv 0$, then the convergence is exponential.

Proof. Let us show that the trajectories enter the invariant set $\Omega(\delta|| \mu||) \times \Sigma_{\varepsilon}(c)$ in finite time. We need now $\delta$ to be small enough so that $s \in \Omega_{s}(\delta|| \mu||)$ and $\varphi \in \Sigma_{\varepsilon}(c)$ imply $\left|\hat{s}_{i}\right|=\mid s_{i}-$ $\varphi_{2 i} \mid<\mu_{i}$ for all $1 \leq i \leq n$. The only place in the proof of Theorem 1, where smallness of $\delta$ causes problems, is the case when $N^{+}(c) \neq \emptyset$ on step (3b), in fact, there is no way to satisfy first two inequalities for $\delta$. Though, in this case one can easily show that $\dot{W} \leq-c_{8}=-\min _{i}\left\{\mu_{i}\left(k_{i}-\right.\right.$ $\left.\left.\gamma_{1 i}(c)\right)\right\}+(n-1) \max _{i}\left\{\tilde{\mu}_{i}(c) \tilde{\gamma}_{1 i}^{2}(c) / k_{i}\right\} / 4$. To make $c_{8}$ positive one have to increase $k_{i}$ 's. With this small correction to the proof of Theorem 1, we conclude that the trajectories enter the invariant set, inside of which none of the components of the control law are saturated.

$$
\text { Let } \quad \tilde{\zeta}=\zeta-\bar{\zeta} \quad \text { and } \quad \tilde{s}=s-\bar{s} \text {. }
$$

Then inside $\Omega(\delta\|\mu\|) \times \Sigma_{\varepsilon}(c)$ (6) could be written as

$$
\begin{aligned}
& \dot{\tilde{\zeta}}=A \tilde{\zeta}+B \tilde{s} \\
& \left.\dot{\tilde{s}}=\tilde{\Delta}(\cdot)-a_{0}(\cdot) K \mu^{-1}\left(\tilde{s}-\varphi_{2}\right)\right) \\
& \varepsilon \dot{\varphi}=A_{f} \varphi+\varepsilon B\left(\tilde{b}_{0}(\cdot)-a_{0}(\cdot) K \mu^{-1}\left(\tilde{s}-\varphi_{2}\right)\right)
\end{aligned}
$$

where $\tilde{b}_{0}(\cdot)=a_{0}(\cdot)\left[-C(\cdot, \cdot)\left(e_{2}+\dot{r}\right)-(g(\cdot)-\right.$ $\left.\left.g\left(q_{d}, \theta\right)\right)\right]-\ddot{r}$ and $\tilde{\Delta}(\cdot)=\tilde{b}_{0}(\cdot)+K_{1} e_{1}+K_{2} e_{2}$.

Clearly, $\left\|\tilde{b}_{0}(\cdot)\right\|,\|\tilde{\Delta}(\cdot)\| \leq \bar{C}_{0}(\bar{\mu})(\|\nu(t)\|+\|\tilde{\zeta}\|+$ $\|\tilde{s}\|)$ for $0<\|\mu\| \leq \bar{\mu}$.

Consider the Lyapunov function

$$
\tilde{V}(\tilde{\zeta}, \tilde{s}, \varphi)=\tilde{\zeta}^{T} P \tilde{\zeta}+\tilde{s}^{T} \tilde{s}+\varphi^{T} P_{f} \varphi
$$

Using the estimates for $\tilde{b}_{0}(\cdot)$ and $\tilde{\Delta}(\cdot)$, it is not hard to show that

$$
\dot{\tilde{V}} \leq-\lambda_{1}(\bar{\varepsilon}, \bar{\mu}) \tilde{V}+\lambda_{2}(\bar{\varepsilon}, \bar{\mu})\|\nu(t)\| \sqrt{\tilde{V}}
$$

for some $\bar{\mu}$ and $\bar{\varepsilon}$, sufficiently small, $0<\|\mu\| \leq$ $\bar{\mu}$, and $0 \leq \varepsilon \leq \bar{\varepsilon}$, where $\lambda_{1}(\cdot)>0, \lambda_{2}(\cdot)>0$.

Obviously, $\tilde{V}(\tilde{\zeta}(t), \tilde{s}(t), \varphi(t)) \rightarrow 0$ as $t \rightarrow \infty$ (exponentially if $\|\nu(t)\| \equiv 0)$. $\tilde{V}$ is a positive definite quadratic form. Hence, $(\tilde{\zeta}(t), \tilde{s}(t), \varphi(t)) \rightarrow$ $(0,0,0)$ as $t \rightarrow \infty$.

Corollary. Suppose $\nu(t) \rightarrow 0$ as $t \rightarrow \infty$ and $k_{i}$ 's can be chosen arbitrarily large. Then, the control law (3), (4), (5) can achieve semiglobal stabilization.

Proof. It is not hard to see that there exists $c$ such that for any given compact set of initial conditions the corresponding initial conditions for (6) are strictly inside the set $\Omega(b) \times \Phi_{\varepsilon}(c)$. If $k_{i}$ 's could be chosen arbitrary large and $\varepsilon$ and $\mu_{i}$ 's could be chosen small enough, then one can always satisfy all the conditions of the theorem.

\section{CONCLUSION}

We have presented a solution for the problems of robust practical semiglobal stabilization and robust set point semiglobal stabilization for directly 
controlled mechanical systems without velocity measurements. The last problem was considered before in (Ortega et al., 1998) and (Pervozvanski and Freidovich, 1999).

It was shown in (Ortega et al., 1998) that the $P I^{2} D$ regulator

$$
\begin{gathered}
u=-k_{p}\left(q-q_{d}\right)-k_{d} b(q-s)-k_{i} v, \\
\dot{s}=\gamma(q-s), \quad \dot{v}=q-q_{d}-b(q-s),
\end{gathered}
$$

where $k_{p}, k_{d}, k_{i}, \gamma$, and $b$ are diagonal matrices with positive elements, semiglobally stabilizes the closed-loop system, provided $\|b\|$ is large enough and $\left\|k_{i}\right\|$ is small enough.

The simple $P I D$ regulator with approximate derivative (Pervozvanski and Freidovich, 1999)

$$
\begin{gathered}
u=-k_{p}\left(q-q_{d}\right)-k_{d}(q-s)-k_{i} v, \\
\dot{s}=\gamma(q-s), \quad \dot{v}=q-q_{d},
\end{gathered}
$$

where $k_{p}, k_{d}, k_{i}$, and $\gamma$ are diagonal matrices with positive elements, also semiglobally stabilizes the closed-loop system ${ }^{2}$.

One disadvantage of using both control laws above is obvious from the singular perturbation analysis of (Pervozvanski and Freidovich, 1999). The smaller the $\left\|k_{i}\right\|$, the slower the convergence of the solutions to the desired equilibrium.

Our regulator (4) also ensures semiglobal asymptotic stabilization but it is based on high gains rather than small ones. Moreover, it can be shown, following the result of (Alvarez-Ramirez et al., 2000 ) and the theory of high-gain observers, that with a special choice of the coefficients $K_{1}, K_{2}$, and $K$ (provided $v(0)=0$ and $\|\mu\|$ is small enough), the performance of the regulator (4) (after a transition period with saturated control) will be as good as that of the ideal inverse dynamics control.

Note also, that regulators (7), (8) must be saturated outside of the domain of interest to be implemented. The controller (4) is decentralized and saturated as usually done in the common industrial practice.

Finally, let us note that the same continuous control law (4) ensures semiglobal practical stabilization when the reference signal is bounded with two bounded derivatives (Theorem 1) and semiglobal asymptotic stabilization when the reference signal is smooth and approaching a given constant limit (Theorem 2), but to guarantee convergence to zero of the tracking error, higher saturation levels are required.

\footnotetext{
2 Though global stabilization is claimed in (Pervozvanski and Freidovich, 1999), what really follows from the proof presented, is semiglobal exponential stabilization. Another way to see this is a direct application of Theorem 3.18 of (Sepulchre et al., 1997).
}

\section{REFERENCES}

Alvarez-Ramirez, J., Cervantes I., Kelly R. (2000), Systems and Control Letters, Vol. 41, 73-83.

Arimoto, S. (1996), Control Theory of Non-linear Mechanical Systems. Clarendon Press, Oxford.

Atassi, N.A. and Khalil, H.K. (1999), IEEE Trans. Aut. Control, Vol. 44, 1672-1687.

Arimoto, S. and Miyazaki, F. (1984), Robotics Research 1. Cambridge: MIT, 783-799.

Berghuis, H. and Nijmeijer,H. (1993), Systems Control Letters, Vol. 21, № 4, 289-293.

Burkov,I.V. (1995), in: Proc. of 2nd Russian Swedish Control Conf., St. Petersburg, Russia, 37-41.

Dunskaya, N.,V. and Pyatnitskii, E.S. (1988), Automat. and Remote Control, $N$ o 12, 40-51.

Freidovich,L.B. (1999), Candidate of Physics and Math. (Ph.D.) thesis, (in Russian), [av. at http://www.math.msu.edu/ leonid/]

Gorez,R. (1999), Systems and Control Letters, Vol. 38, 61-72.

Hoppensteadt, F. (1974), J. Differ. Equations, Vol. 15, $N^{o}$ 3, 510-521.

Isidori, A. (1995), Nonlinear control systems, 3rd edition, N.Y.: Springer-Verlag.

Kelly, R. (1998), IEEE Trans. on Aut. Control, Vol. 43, $N^{o}$ 7, 934-937.

Khalil,H.K. (1996), Nonlinear systems, 2nd ed., Prentice Hall, Upper Saddle River, NJ.

Khalil, H.K. (2000), IEEE Trans. on Aut. Control, Vol. 45, No 3, 490-494.

Loria, A., Kelly, R., Ortega, R., and Santibañez, V. (1996), IEEE Trans. on Aut. Control, Vol. 42, No 8, 1138-1142.

Meressi, T., Chen, D., and Paden, B. (1993), IEEE Trans. on Aut. Control, Vol. 38, No 3, 488491.

Ortega, R., Loria, A., Sira-Ramirez, H., and Nicklasson, P.J. (1998), Passivity-based Control of Euler-Lagrange Systems, Springer Verlag.

Pervozvanski,A. A. and Freidovich,L.B. (1999), Dynamics and Control, $N^{o}$ 9, 203-222.

$\mathrm{Qu}, \mathrm{Z}$. and Dorsey, J. (1991), Int. J. Robotic and Automat., Vol. 6, № 4, 228-235.

Rocco,P. (1996), IEEE Trans. on Robot. and Aut., Vol. 12, $N$ o 4, 607-614.

Santibañez, V., and Kelly, R. (1998), in: Proc. IEEE Int. Conf. on Robotics and Automation, 3601-3606.

Sepulchre,R., Jankovic,M., and Kokotovic, P. (1997), Constructive Nonlinear Control, Springer Verlag, London.

Spong, M.W. and Vidyasagar, M. (1989), Robot Dynamics and Control, Wiley, NY.

Takegaki, M. and Arimoto, S. (1981), Trans. ASME J. of Dynam. Syst. Meas. Control, Vol. 103, 119-125.

Tomei, P. (1991), IEEE Trans. on Robot. Aut., Vol. 7, No $4,565-570$. 